\title{
Factores de riesgo asociados al diagnóstico de alergia al huevo IgE mediada en Pediatría
}

\author{
Risk factors associated with the diagnosis of IgE-mediated egg \\ allergy in Pediatrics
}

\author{
Viviana Sotomayor ${ }^{1}$, Marta Cristina Sanabria'
}

\section{RESUMEN}

Introducción: La prevalencia de alergias alimentarias se ha incrementado, lo que representa un problema de salud pública. Las guías alimentarias recomendaban retrasar la introducción de alimentos de manera a prevenir alergias alimentarias sin real evidencia. Objetivos: Describir el perfil clínico del paciente pediátrico que acude a consulta con el diagnóstico de alergia al huevo. Materiales y métodos: Estudio de observacional retrospectivo descriptivo de. Ingresaron al estudio pacientes con sospecha de alergia al huevo que tuvieron estudio de RAST. Se estudio datos demográficos, lactancia materna, inicio de alimentación solida incluyendo el huevo, presencia de manisfestaciones de alergia. Se realizo comparación entre los grupo con RAST positivo y aquellos con resultados negativo. Los datos fueron procesados en Microsoft Excell y se utilizó variables descriptivas. Resultados: ingresaron 156 pacientes ,58 en cada grupo con prueba positiva y negativa. Los pacientes alérgicos al huevo se presentaron con un predominio de sexo masculino, con antecedentes personales de atopia, peso y tallas normales, duración de la lactancia materna promedio de 10,5 meses, introducción de sólidos a los 5,7 meses, edad de ingesta de huevo a los 9,2 meses, similar al grupo con test negativo. Los síntomas predominantes fueron los gastrointestinales Como manifestación de atopia concomitante tuvieron mayor porcentaje de Alergia a las proteínas de leche de vaca y dermatitis atópica en comparación con el grupo con test negativo. Conclusiones: No hubo diferencias demograficas ni de habitos alimentarios entre los grupos con RAST postivo y negativo Hubo concomitancia de otras manisfestaciones de alergia en el grupo RAST postivo.

Palabras claves: Alergia al huevo, lactancia materna, síntomas gastrointestinales, alimentación.

\section{ABSTRACT}

Introduction: The prevalence of food allergies has increased, representing a public health problem. Current dietary guidelines recommend delaying the introduction of food in order to prevent food allergies, but supporting evidence for this is lacking. Objectives: To describe the clinical profile of pediatric patients presenting with a diagnosis of egg allergy. Materials and methods: This was a descriptive, retrospective, observational study. We included patients with a suspected egg allergy who had a RAST study. We evaluated demographic data, breastfeeding, introduction of solid foods, including egg, and the presence of allergy manifestations. A comparison was made between the groups with positive RAST and those with negative results. The data was processed in Microsoft Excel and descriptive variables were used. Results: 156 patients were included in the study, 58 in each group with positive and negative tests. Egg-allergic patients were predominantly male, with a personal history of atopy, normal weight and size, an average breastfeeding duration of 10.5 months, introduction of solids at 5.7 months, age of intake of egg at 9.2 months, which was similar to the group with negative testing. The predominant symptoms were gastrointestinal. Concomitant atopy manifestations included a higher percentage of cow's milk protein allergy and atopic dermatitis compared to the group with a negative testing. Conclusions: There were no demographic or dietary differences between the groups with positive and negative RAST. There was concomitance of other allergic manifestations in the positive RAST group.

Keywords: Egg allergy, breastfeeding, gastrointestinal symptoms, feeding.

${ }^{1}$ Universidad Nacional de Asunción. Cátedra y Servicio de Pediatría. San Lorenzo, Paraguay.

Correspondencia: Viviana Sotomayor Correo: dravivianasotomayor@gmail.com

Conflicto de interés: Los autores declaran no poseer conflicto de interés

Recibido:7/07/2019 Aceptado:23/07/2019

DOI: https://doi.org/10.31698/ped.46022019007






\section{INTRODUCCIÓN}

El huevo es una fuente de alimentación importante en nuestro medio, incluyendo un rango variable de productos alimentarios manufacturados. Es indispensable conocer el comportamiento clínico de la alergia al huevo para no suspender innecesariamente su consumo y conocer la forma de reintroducirlo de nuevo a la dieta, seleccionando pacientes adecuadamente, pues es una fuente de aminoácidos esenciales en la nutrición del niño y de adquisición de proteínas de alto valor biológico ${ }^{(1)}$.

Las guías alimentarias para menores de dos años, durante mucho tiempo recomendaron retrasar la introducción de alimentos y potenciales alergenos alimentarios en grupos de riesgo sin reales evidencias $^{(2)}$.

Aunque la alergia al huevo en sí puede ser superada, estos niños tienen un mayor riesgo de otras enfermedades atópicas posteriores, como el asma y la rinitis alérgica, y algunos presentan a la vez otras alergias alimentarias que persisten en la vida adulta, como alergia al maní y nueces ${ }^{(3-5)}$. El objetivo del presente estudio fue describir el perfil clínico del paciente pediátrico con alergia al huevo.

\section{MATERIALES Y MÉTODOS}

Diseño, estudio de observacional retrospectivo transversal, con componente analítico. Con muestreo no probabilístico de casos consecutivos, ingresaron al estudio pacientes pediátricos con sospecha de alergia al huevo, con estudios de RAST (prueba de radio alergo absorbencia) específico para huevo, que consultaron en la Cátedra y Servicio de Pediatría, Facultad de Ciencias Médicas. Universidad Nacional de Asunción (FCM-UNA), del año 2000 a 2016.Se estudiaron las variables edad, sexo, modo de nacimiento, estado nutricional, antecedentes familiares de atopia, comorbilidades y síntomas asociados, duración de la lactancia materna, edad de introducción de los alimentos sólidos en general y del huevo en particular, dosaje de IGE total. De acuerdo a este resultado se formó 2 grupos, los que tenía la prueba positiva y aquellos con prueba negativa.

El cálculo del estado nutricional se realizó como P/E para los menores de 2 años y $\mathrm{P} / \mathrm{T}$ para los de $\geq 2$ años de edad, y la interpretación de acuerdo a la curva de OMS.

Los datos fueron procesados en el programa Microsoft Excel 2013.Se utilizaron estadísticas descriptivas,las variables cualitativas se expresaron en porcentajes y las cuatitativas como medias o medianas de acuerdo a su distribución.

\section{RESULTADOS}

Fueron incluidos 116 pacientes 58 con alergia al huevo con RAST positivo y 58 pacientes en quienes no se confirmó alergia por RAST negativo.

Los datos demográficos, antecedentes familiares de atopia, estado nutricional y nacimiento por cesárea de cada grupo se encuentran en la Tabla 1.

Tabla 1. Caracteriticas de los grupos de pacientes con sospecha de alergia al huevo y según resultados de RAST N=116.

\begin{tabular}{llll}
\hline Variables & $\begin{array}{l}\text { Grupo RAST (-) } \\
\mathbf{n}=\mathbf{5 8}\end{array}$ & $\begin{array}{l}\text { Grupo RAST (+) } \\
\mathbf{n}=\mathbf{5 8}\end{array}$ & $\mathbf{P}$ \\
\hline $\begin{array}{l}\text { Edad (meses) } \\
\text { mediana }\end{array}$ & 28 & 24 & \\
$\begin{array}{l}\text { Sexo masculino } \\
\text { N }(\%)\end{array}$ & $36(62)$ & $37(63,8)$ & $0,85^{*}$ \\
$\begin{array}{l}\text { Antecedentes familiares } \\
\text { de atopia }\end{array}$ & $42(72,4)$ & $48(82,7)$ & $0,26^{*}$ \\
$\begin{array}{l}\text { Estado nutricional } \\
\text { P/E: Eutroficos }\end{array}$ & $30(51,7)$ & $32(55)$ & $0,85^{*}$ \\
\hline $\begin{array}{l}\text { Estado nutricional } \\
\text { T/E :Eutroficos }\end{array}$ & $52(90)$ & $54(93)$ & $0,14 \mathrm{~F}^{* *}$ \\
$\begin{array}{l}\text { Nacimiento por } \\
\text { cesárea }\end{array}$ & $30(51,7)$ & $29(50)$ & $0,85^{*}$ \\
\hline *chi cuadrado **Test de Fisher & & &
\end{tabular}

Se analizaron algunos aspectos de la alimentación y la presencia de comorbilidades alérgicas, los resultados se muestran en la Tabla 2.

Los pacientes que presentaron comorbilidades alérgicas el 72,2\% (13/18) presentaban dermatitis atópica y concomitantemente el mismo porcentaje de pacientes presentaron alergia a las proteínas de leche de vaca (APLV).

Se analizaron los principales síntomas clínicos en ambos grupos de pacientes con los resultados expresado en la Tabla 3. 
Tabla 2. Algunos aspectos de la alimentación y presencia de cormorbilidades en ambos grupos de pacientes $\mathrm{N}=116$.

\begin{tabular}{llll}
\hline Variables & $\begin{array}{l}\text { Grupo RAST (-) } \\
\mathbf{n = 5 8}\end{array}$ & $\begin{array}{l}\text { Grupo RAST (+) } \\
\mathbf{n = 5 8}\end{array}$ & $\mathbf{P}$ \\
\hline $\begin{array}{l}\text { Duración de lactancia } \\
\text { materna (meses) mediana }\end{array}$ & 10,5 & 10,1 & 0,69 \\
\hline $\begin{array}{l}\text { Edad de inicio de sólidos } \\
\text { (meses) mediana }\end{array}$ & 5,7 & 5,8 & 0,26 \\
\hline $\begin{array}{l}\text { Edad de introducción del } \\
\text { huevo(meses) mediana }\end{array}$ & 9,2 & 9,8 & 0,69 \\
$\begin{array}{l}\text { Co morbilidades alérgicas } \\
\mathbf{N}(\%)\end{array}$ & $18(31)$ & $13(22,4)$ & 0,40 \\
$\begin{array}{l}\text { Co morbilidades no } \\
\text { alérgicas } \mathbf{N}(\%)\end{array}$ & $9(15,5)$ & $4(7)$ & 0,23 \\
\hline
\end{tabular}

Tabla 3. Síntomas clínicos en los grupos de pacientes estudiados $\mathrm{N}=116$.

\begin{tabular}{llll}
\hline Tipo de síntomas & $\begin{array}{l}\text { Grupo RAST (-) } \\
\mathbf{n = 5 8}\end{array}$ & $\begin{array}{l}\text { Grupo RAST (+) } \\
\mathbf{n = 5 8}\end{array}$ & $\mathbf{P}$ \\
\hline & $\mathrm{N}(\%)$ & $\mathrm{N}(\%)$ & \\
Cutáneos & $35(60,3)$ & $38(65,5)$ & $0,70^{*}$ \\
$\quad$ - Urticaria & $34(97)$ & $34(97)$ & \\
$\quad$ - angioedema & $8(23,5)$ & $8(13,8)$ & $0,67^{*}$ \\
Respiratorios & $41(70,7)$ & $44(75,8)$ & \\
Tos chillido & $36(87,8)$ & $44(100)$ & $0,03^{*}$ \\
Rinitis & $31(75,6)$ & $36(81.8)$ & \\
Digestivos & $19(32,7)$ & $9(15,5)$ & \\
Diarrea & $12(63)$ & $9(100)$ & \\
Distención abdominal & $9(47,3)$ & 0 & \\
Vómitos & $8(42,1)$ & $2(22,2)$ & \\
Reflujo GI & $5(26,3)$ & $2(22,2)$ & \\
Constipación & $3(15,7)$ & $2(22,2)$ & \\
\hline
\end{tabular}

*chi cuadrado

\section{DISCUSIÓN}

Este trabajo la mayoría de los niños en ambos grupos presentaron como mediana de ingesta de huevo la edad de nueve meses, esto puede justificarse además por las costumbres familiares y también por la distinta aceptación de los médicos a las guías alimentarias. Esta falta de relación aparentemente existente entre la edad de introducción del huevo y la alergia al huevo apoyaría las nuevas guías alimentarias donde se recomienda no postergar la introducción del huevo en la dieta. $\mathrm{Al}$ respecto, un estudio realizado en lactantes chilenos, que evaluó la relación entre el patrón alimentario y la presencia de manifestaciones alérgicas, no encontró ninguna diferencia en la temporalidad de inclusión de los alimentos sólidos entre lactantes alérgicos y controles sanos, con un cumplimiento considerable de los tiempos de inclusión recomendados (después de los 6 meses de edad) en ambos grupos ${ }^{(7,8)}$ A su vez, Zutavern y $\operatorname{cols}^{(9)}$., en un estudio prospectivo de cohorte de nacimiento mostraron un aumento del riesgo de eczema relacionado con la introducción tardía de huevos y leche. La introducción tardía de huevo mostró una tendencia a aumentar el riesgo de sibilancias en la edad preescolar. En el estudio se encontró que, en comparación con la introducción del huevo entre los 4 y 6 meses, la ingesta más tardía del mismo se asoció a riesgo más elevado de alergia al mismo (ORs], 1.6 [95\% CI, 1.0-2.6] y 3.4 [95\% CI, 1.8-6.5] para la introducción tardía). Por otro lado, una reciente investigación que evaluó la exposición temprana y regular al huevo en lactantes con eczema atópico, mostró que al año el grupo que recibió huevo tempranamente tuvo una menor frecuencia de diagnóstico de alergia mediada por $\operatorname{Ig} \mathrm{E}^{(10)}$.

Hasta hace poco, las guías alimentarias de varios países recomendaban retardar la introducción de alérgenos alimentarios (retardando la introducción de huevo hasta los un año y nueces hasta los tres años) en lactantes con historia familiar de alergia; también se aconsejaba retardar la alimentación sólida hasta los 6 meses y continuar con lactancia materna hasta el año de vida ${ }^{(5)}$. Estas recomendaciones han sido reevaluadas en estudios poblacionales recientes, sustentando que la práctica de atrasar la introducción de ciertos alérgenos aumenta más que disminuye el riesgo de alergia ${ }^{(5,6)}$.

El impacto de factores alimentarios en el desarrollo y mantención de la tolerancia oral es un área de investigación emergente en la cual los mecanismos moleculares involucrados no son del todo $\operatorname{conocidos}^{(10,1)}$. Sin embargo, es claro que el efecto beneficioso en la modulación del sistema inmune del lactante guarda estrecha relación con el momento en el cual son incorporados en la dieta, tanto materna como infantil. Por tanto, las prácticas alimentarias durante el período perinatal y los primeros meses de la vida parecen ser relevantes en el desenlace futuro de un desorden alérgico ${ }^{(8,12)}$. Los primeros meses de vida representan una fase crítica en el desarrollo de la tolerancia oral, momento en el cual la microbiota no está completamente establecida y la barrera epitelial aun está en desarrollo ${ }^{(13)}$. La alimentación 
láctea, ya sea como LM o FL artificiales y el momento en el cual son incorporados los alimentos sólidos en la dieta del lactante, representan importantes factores ambientales posibles de ser modulados y que podrían contribuir a la mantención de una respuesta inmune adecuada ${ }^{(9,13)}$.

\section{CONCLUSION}

\section{REFERENCIAS BIBLIOGRÁFICAS}

1. Sicherer SH, Sampson HA. Food allergy: recent advances in pathophysiology and treatment. Annu Rev Med. 2009; 60:261-77.

2. Sicherer SH. Epidemiology of food allergy. J Allergy Clin Inmunol. 2011; 127:594-602.

3. Branum AM, Lukacs SL. Food Allergy among children in the United States. Pediatrics. 2009; 124:1549-55.?

4. Sicherer SH, Munoz-Furlong A, Godbold JH, Sampson HA. US Prevalence of self-reported peanut, tree nut and sesame allergy: 11-year follow-up. J Allergy Clin Immunol. 2010;125:1322-6.

5. Gupta, Sheik A, Strachan DP, Anderson HR. Time Trends in allergic disorders in the UK. Thorax 2007; 62: 91-6.

6. Sicherer SH, Sampson HA. Food allergy: recent advances in pathophysiology and treatment. Annu Rev Med. 2009; 60:261-77.

7. Bascuñán KA, Weisstaub G, Chamorro R, Guzmán M, Araya M. Asociación entre los patrones alimentarios durante el primer año de vida y alergia alimentaria en lactantes. Arch Argent Pediatr. 2012;110:375-81.
No hubo diferencias demográficas ni de hábitos alimentarios entre los grupos con RAST positivo y negativo Hubo concomitancia de otras manifestaciones de alergia en el grupo RAST positivo. Los síntomas digestivos predominaron en l grupo con RAST positivo.

8. Rona RJ, Keil T, Summers C, Gislason D, Madsen C, Summer C, et al. The prevalence of food allergy: a metaanalysis. J Allergy Clin Immunol. 2007; 120:638-46.

9. Zutavern A, Brockow I, Schaaf B, Bolte G, von Berg A, Diez $U$, et al. Timing of solid food introduction in relation to atopic dermatitis and atopic sensitization: results from a prospective birth cohort study. Pediatrics. 2006; 117:401-11.

10. Bascuñán K, Araya M. Potenciales intervenciones alimentarias en el manejo y prevención de la alergia en lactantes. Nutr Hosp. 2014; 29(5):969-978.

11. Diéguez MC, Cerecedo I, Muriel A, Zamora J, Abraira V, Camacho E, et al. Utility of diagnostic tests in the follow-up of egg-allergic children. Clin Exp Allergy. 2009; 39(10):1575-84.

12. Nwaru BI, Erkkola M, Ahonen S, Kaila M, Haapala AM, Kronberg-Kippila C, et al. Age at the introduction of solid foods during the first year and allergic sensitization at age 5 years. Pediatrics. 2010; 125:50-9.

13. Round JL, Mazmanian SK. Inducible Foxp3+ regulatory T-cell development by a commensal bacterium of the intestinal microbiota. Proc Natl Acad Sci U S A. 2010; 107(27):12204-12209. 